\title{
Gapless topological Fulde-Ferrell superfluidity induced by an in-plane Zeeman field
}

\author{
Hui Hu, ${ }^{1}$ Lin Dong, ${ }^{2}$ Ye Cao, ${ }^{1}$ Han Pu, ${ }^{2}$ and Xia-Ji Liu ${ }^{1}$ \\ ${ }^{1}$ Centre for Quantum and Optical Science, Swinburne University of Technology, Melbourne 3122, Australia \\ ${ }^{2}$ Department of Physics and Astronomy, and Rice Quantum Institute, Rice University, Houston, Texas 77251, USA
}

(Received 14 August 2014; published 24 September 2014)

\begin{abstract}
Topological superfluids are recently discovered quantum matter that hosts topologically protected gapless edge states known as Majorana fermions-exotic quantum particles that act as their own antiparticles and obey non-Abelian statistics. Their realizations are believed to lie at the heart of future technologies such as fault-tolerant quantum computation. To date, the most efficient scheme to create topological superfluids and Majorana fermions is based on the Sau-Lutchyn-Tewari-Das Sarma model with a Rashba-type spin-orbit coupling on the $x-y$ plane and a large out-of-plane (perpendicular) Zeeman field along the $z$ direction. Here we propose an alternative setup, where the topological superfluid phase is driven by applying an in-plane Zeeman field. This scheme offers a number of different features, notably Cooper pairings at finite center-of-mass momentum (i.e., Fulde-Ferrell pairing) and gapless excitations in the bulk. As a result, gapless topological quantum matter with an inhomogeneous pairing order parameter appears. It features unidirectional Majorana surface states at boundaries, which propagate in the same direction and connect two Weyl nodes in the bulk. We demonstrate the emergence of such exotic topological matter and the associated Majorana fermions in spin-orbit coupled atomic Fermi gases, and we determine its parameter space. The implementation of our scheme in semiconductor/superconductor heterostructures is briefly discussed.
\end{abstract}

DOI: 10.1103/PhysRevA.90.033624

PACS number(s): 03.75.Hh, 05.30.Fk, 03.75.Ss, 67.85.-d

\section{INTRODUCTION}

The possibility of realizing topological superfluids and manipulating Majorana fermions in solid-state and ultracold atomic systems is currently a topic of great theoretical and experimental interest [1,2], due to their fundamental interest and potential applications in fault-tolerant topological quantum computation [3]. Roughly, Majorana fermions constitute "half" of an ordinary Dirac fermion, in the sense that two real Majorana fermions $\gamma_{1}$ and $\gamma_{2}$-which can be separated in arbitrary distance-mathematically define a complex fermion operator $c=\gamma_{1}+i \gamma_{2}$ [4]. The exchange statistics of Majorana fermions is exotic. Unlike conventional bosons and fermions, braiding Majorana fermions around one another in a $2^{N}$ dimensional Hilbert space (spanned by $2 N$ well-separated Majorana fermions) produces non-Abelian unitary transformations in the Hilbert space. Quantum information can then be nonlocally encoded in the Hilbert space by such braiding operators and be immune to decoherence, which is ideal for the purpose of quantum computation [3].

At present, a number of experimental settings have been suggested for hosting Majorana fermions under appropriate conditions, including chiral $p$-wave superconductors [5], fractional quantum Hall systems at filling $v=5 / 2$ [6], and topological insulators or semiconductor nanowires in proximity to an $s$-wave superconductor [7-9]. The latest setting, which seems to be the most practical setup, is described by the Sau-Lutchyn-Tewari-Das Sarma (SLTD) model [8]. The key idea of this mechanism is that the Fermi surfaces are spin-split by a Rashba spin-orbit coupling in the $x-y$ plane and a perpendicular out-of-plane Zeeman field along the $z$ direction. If the number of particles is tuned to make the inner Fermi surface disappear, superconductivity will only be induced by pairing on the outer Fermi surface, which is $p$-wave in nature $[10,11]$, and therefore it becomes topologically nontrivial. Following this promising theoretical model, exciting experimental progress for the observation of Majorana fermions has been made very recently [12-14], although unambiguous confirmation for their existence still remains elusive.

The SLTD mechanism uses an out-of-plane Zeeman field to split the Fermi surfaces [8]. It is interesting that such a splitting can also be achieved by applying a large inplaneZeeman field in combination with spin-orbit coupling, as illustrated in Fig. 1. Furthermore, the in-plane field together with spin-orbit coupling is known to introduce an asymmetry in the single-particle dispersion $[15,16]$, and consequently to induce Cooper pairs with nonzero center-of-mass momentum [17-33] and hence realize the so-called spatially inhomogeneous Fulde-Ferrell (FF) pairing scenario [34]. It is therefore of interest to ask whether a topological phase transition can also be driven by an in-plane Zeeman field only? If the answer is yes, then we must be able to observe an exotic inhomogeneous topological FF superfluid that supports Majorana fermions. The understanding of such a topological state of matter may greatly enrich our knowledge about topological superfluids.

In this work, we examine the mechanism by using an ultracold atomic setting of a three-dimensional (3D) spinorbit-coupled atomic Fermi gas subject to an in-plane Zeeman field. By increasing the field strength above a threshold, we observe the change of the topology of the Fermi surfaces that triggers a topological phase transition. The resulting inhomogeneous topological FF superfluid is gapless in the bulk with nodal points forming closed surfaces in momentum space, while in real space it hosts unidirectional Majorana surface states that propagate in the same direction at boundary. These unique features are absent in standard topological superfluids known so far, such as the $p$-wave superconductors or the SLTD-type superconductors, both of which are gapped in the bulk and support counterpropagating Majorana modes at 


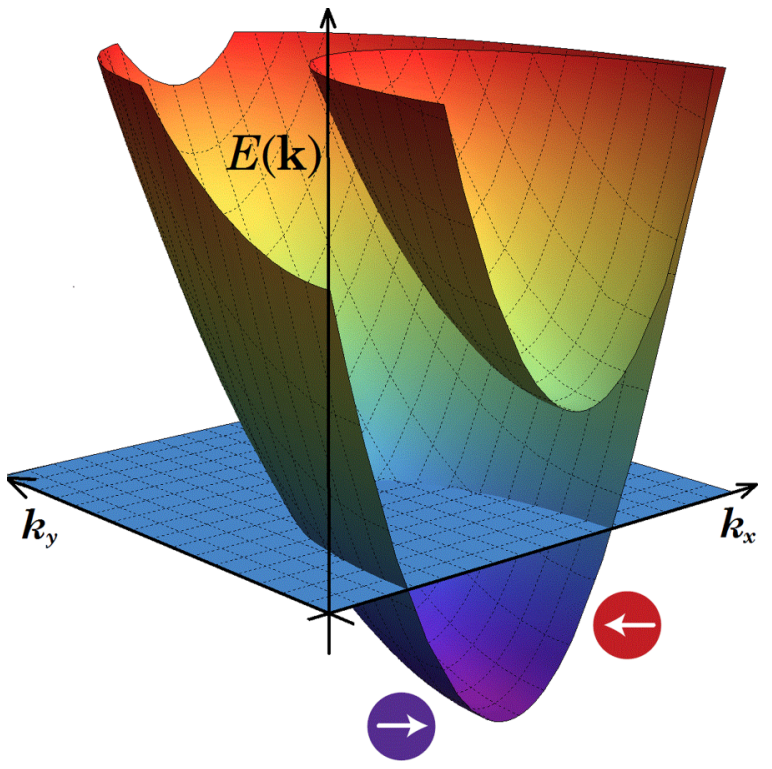

FIG. 1. (Color online) Formation of an effective $p$-wave energy band. The energy band of the system splits into two by spin-orbit coupling. A large in-plane Zeeman field along the $x$ direction strongly tilts the energy dispersion. When the Fermi energy lies below the upper band, atoms occupy the lower band only and form an effective "spinless" system, in which the composite particle consists of both spin-up and spin-down ingredients (shown by circles with arrows), and they interact with each other via an effective $p$-wave interaction.

surfaces. We find that the phase space for the proposed gapless topological FF superfluid is significant, implying that it could be easily realized in current ultracold atomic experiments due to the unprecedented tunability of synthetic spin-orbit coupling, the Zeeman field, and interatomic interaction in a cold-atom laboratory [35-38]. We also discuss briefly the potential implementation of our proposal in solid-state setups.

We note that the possibility of using an in-plane Zeeman field to induce a topological transition has been explored earlier by Alicea [39] as a variation of the SLTD mechanism. The key idea of that work is to use both Dresselhaus and Rashba spin-orbit couplings, which in a certain limit recovers the SLTD model. The FF pairing induced by the in-plane Zeeman field is not discussed. The gapless superfluid is clearly regarded as topologically trivial. On the other hand, the system that we shall investigate below has been considered in earlier works $[25,26]$. However, in these works the possibility of topological superfluidity is not realized, and the associated nontrivial unidirectional Majorana edge states are not discussed.

\section{MODEL HAMILTONIAN AND MEAN-FIELD THEORY}

For concreteness, we focus on a 3D spin-orbit-coupled two-component Fermi gas with an isotropic spin-orbit coupling $V_{\mathrm{SO}}(\hat{\mathbf{k}})=\lambda\left(\hat{k}_{x} \sigma_{x}+\hat{k}_{y} \sigma_{y}+\hat{k}_{z} \sigma_{z}\right)$ subject to an in-plane Zeeman field $h \sigma_{x}[25,26,40-42]$. This is probably the simplest system to demonstrate the effect of an in-plane Zeeman field, because, with the spin-orbit coupling expanding in all three directions, all the Zeeman fields should be classified as in-plane. Thus, we avoid any possible complications due to the existence of an out-of-plane Zeeman field. In cold-atom experiments, the creation of a 3D isotropic spin-orbit coupling or a 2D Rashba spin-orbit coupling may be achieved by a sequence of pulsed inhomogeneous magnetic fields that imprint suitable phase gradients on the atoms, with similar experimental difficulty [41,42].

The above-mentioned Fermi gas with isotropic spin-orbit coupling can be described by the model Hamiltonian,

$$
\mathcal{H}=\int d \mathbf{r}\left[\sum_{\sigma \sigma^{\prime}} \psi_{\sigma}^{\dagger}(\mathbf{r}) \mathrm{H}_{0}^{\sigma \sigma^{\prime}} \psi_{\sigma^{\prime}}(\mathbf{r})+\mathcal{V}_{\mathrm{int}}\right],
$$

where $\psi_{\sigma}^{\dagger}(\mathbf{r})\left(\psi_{\sigma}\right)$ is the field operator for creating (annihilating) an atom with pseudospin state $\sigma \in(\uparrow, \downarrow)$ at position $\mathbf{r}$, $H_{0}=-\hbar^{2} \nabla^{2} /(2 m)-\mu+V_{\text {SO }}(\hat{\mathbf{k}})+h \sigma_{x}$ is the single-particle Hamiltonian with the atomic mass $m$ and chemical potential $\mu, \hat{k}_{i=(x, y, z)}=-i \partial_{i}$ is the momentum operator, and $\sigma_{x, y, z}$ are the Pauli matrices. $\mathcal{V}_{\text {int }}=U_{0} \psi_{\uparrow}^{\dagger}(\mathbf{r}) \psi_{\downarrow}^{\dagger}(\mathbf{r}) \psi_{\downarrow}(\mathbf{r}) \psi_{\uparrow}(\mathbf{r})$ describes a pairwise attractive contact interaction of strength $U_{0}<0$, where $U_{0}^{-1}=m /\left(4 \pi \hbar^{2} a_{s}\right)-V^{-1} \sum_{\mathbf{k}} m /\left(\hbar^{2} \mathbf{k}^{2}\right)$ can be expressed in terms of the $s$-wave scattering length $a_{s}$. At the mean-field level, the model Hamiltonian can be solved by taking an order parameter $\Delta(\mathbf{r})=-U_{0}\left\langle\psi_{\downarrow}(\mathbf{r}) \psi_{\uparrow}(\mathbf{r})\right\rangle$ and linearizing the interaction Hamiltonian $\mathcal{V}_{\text {int }} \simeq-\left[\Delta(\mathbf{r}) \psi_{\uparrow}^{\dagger}(\mathbf{r}) \psi_{\downarrow}^{\dagger}(\mathbf{r})+\right.$ H.c.] $-|\Delta(\mathbf{r})|^{2} / U_{0}$.

In the presence of an in-plane Zeeman field $h \sigma_{x}$, it is now widely understood that Cooper pairs acquire a finite center-of-mass momentum $\mathbf{Q}=q \mathbf{e}_{x}$ along the $x$ direction, i.e., $\Delta(\mathbf{r})=\Delta e^{i q x}[15,22-25]$. This helical phase was first proposed in the context of noncentrosymmetric superconductors [17-21,43]. By using the Nambu spinor $\Phi(\mathbf{r}) \equiv$ $\left[\psi_{\uparrow} e^{+i q x / 2}, \psi_{\downarrow} e^{+i q x / 2}, \psi_{\uparrow}^{\dagger} e^{-i q x / 2}, \psi_{\downarrow}^{\dagger} e^{-i q x / 2}\right]^{T}$ to gauge out the momentum-related phase in the order parameter, the meanfield model Hamiltonian can be solved by diagonalizing the following Bogoliubov-de Gennes (BdG) Hamiltonian:

$$
\mathcal{H}_{\mathrm{BdG}}(\hat{\mathbf{k}}) \equiv\left[\begin{array}{cc}
H_{0}\left(\frac{\mathbf{Q}}{2}+\hat{\mathbf{k}}\right) & -i \Delta \sigma_{y} \\
i \Delta \sigma_{y} & -H_{0}^{*}\left(\frac{\mathbf{Q}}{2}-\hat{\mathbf{k}}\right)
\end{array}\right]
$$

i.e., $\mathcal{H}_{\mathrm{BdG}} \Phi_{\mathbf{k} \eta}^{\nu}(\mathbf{r})=E_{\eta \nu}(\mathbf{k}) \Phi_{\mathbf{k} \eta}^{v}(\mathbf{r})$, which gives rise to the wave function of Bogoliubov quasiparticles, $\Phi_{\mathbf{k} \eta}^{v}(\mathbf{r})=$ $1 / \sqrt{V} e^{i \mathbf{k} \cdot \mathbf{r}}\left[u_{\eta \uparrow}^{v}, u_{\eta \downarrow}^{v}, v_{\eta \uparrow}^{v}, v_{\eta \downarrow}^{\nu}\right]^{T}$, and the energy $E_{\eta \nu}(\mathbf{k})$. We obtain four quasiparticle energy dispersions, indexed by $v \in$ $(+,-)$ for the particle $(+)$ or hole $(-)$ branch, and $\eta \in(1,2)$ for the upper (1) or lower (2) band split by the spin-orbit coupling and Zeeman field. We derive the gap and number equations from the resulting mean-field thermodynamic potential (see Appendix A) and solve them self-consistently to obtain $\Delta$, $q$, and $\mu$, from which we determine the phase diagram at zero temperature. In our numerical calculations, using the number density $n$ we have set the Fermi wave vector $k_{F}=$ $\left(3 \pi^{2} n\right)^{1 / 3}$ and Fermi energy $E_{F}=\hbar^{2} k_{F}^{2} /(2 m)$ as the units for wave vector and energy, respectively. Unless specifically noted, we shall focus on the weak-coupling case with a dimensionless interaction parameter $1 /\left(k_{F} a_{s}\right)=-0.5$ and at zero temperature $T=0$, for which our mean-field treatment could be well justified. 


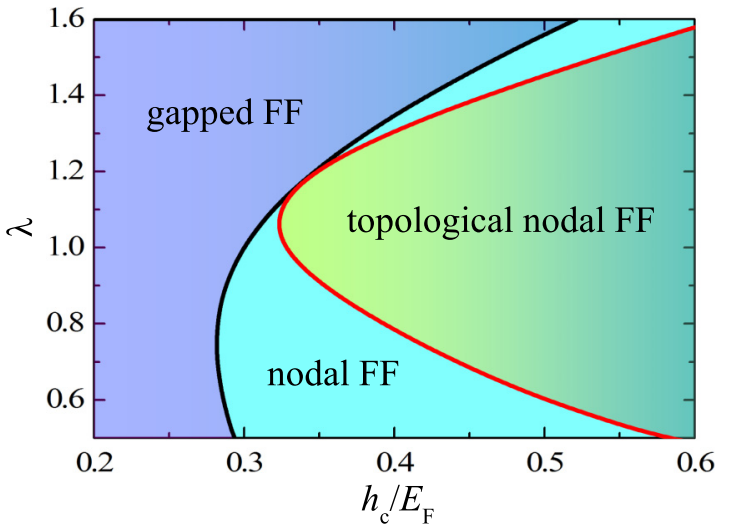

FIG. 2. (Color online) Zero-temperature phase diagram of the FF superfluid at the interaction parameter $1 /\left(k_{F} a_{s}\right)=-0.5$. With an increasing in-plane Zeeman field, the Fermi cloud changes from a gapped FF superfluid to a gapless FF superfluid, and finally it turns into a gapless topological superfluid. The strength of spin-orbit coupling is in units of $E_{F} / k_{F}$.

\section{RESULTS AND DISCUSSIONS}

\section{A. Zero-temperature phase diagram}

In Fig. 2, we report the zero-temperature phase diagram. It is readily seen from the phase diagram that an in-plane Zeeman field will drive the Fermi system from a gapped FF superfluid to a gapless phase (labeled as "nodal FF") [25,26]. Remarkably, at a sufficiently large value it will also lead to a gapless topologically nontrivial state ("topological nodal FF") $[32,33]$. The evolution of the energy spectrum at a typical spinorbit coupling strength $\lambda=E_{F} / k_{F}$ as a result of the increasing in-plane Zeeman field is presented in Fig. 3.

Physically, the transition to a gapless phase can be well characterized by a global energy gap $E_{g}=\min E_{2+}(\mathbf{k})$, which is half of the energy difference between the minimum energy of the particle branch and the maximum of the hole branch due to the particle-hole symmetry $E_{2+}(\mathbf{k})=-E_{2-}(-\mathbf{k})$. Hence, $E_{g} \leqslant 0$ and $E_{g}>0$ characterize a gapless and gapped state, respectively. The topological phase transition, on the contrary, is related to the change of the topology of the Fermi surfaces. It is well known that such a change must be accompanied by the closing and reopening of an energy gap at some specific points in momentum space $[1,2]$. In our continuum case of a homogeneous Fermi gas, this occurs precisely at the origin $\mathbf{k}=\mathbf{0}$ [see Fig. 3(b)]. Therefore, naively the topological transition can be determined from the condition $E_{2+}(\mathbf{k}=\mathbf{0})=0$, or more explicitly [30,32],

$$
h_{c 2}=\sqrt{\left(\mu-\frac{\hbar^{2} q^{2}}{8 m}\right)^{2}+\Delta^{2}}-\frac{\lambda q}{2} .
$$

In the absence of an FF pairing momentum $(q=0)$, the above condition reduces to the well-known criterion $h_{c}=\sqrt{\mu^{2}+\Delta^{2}}$
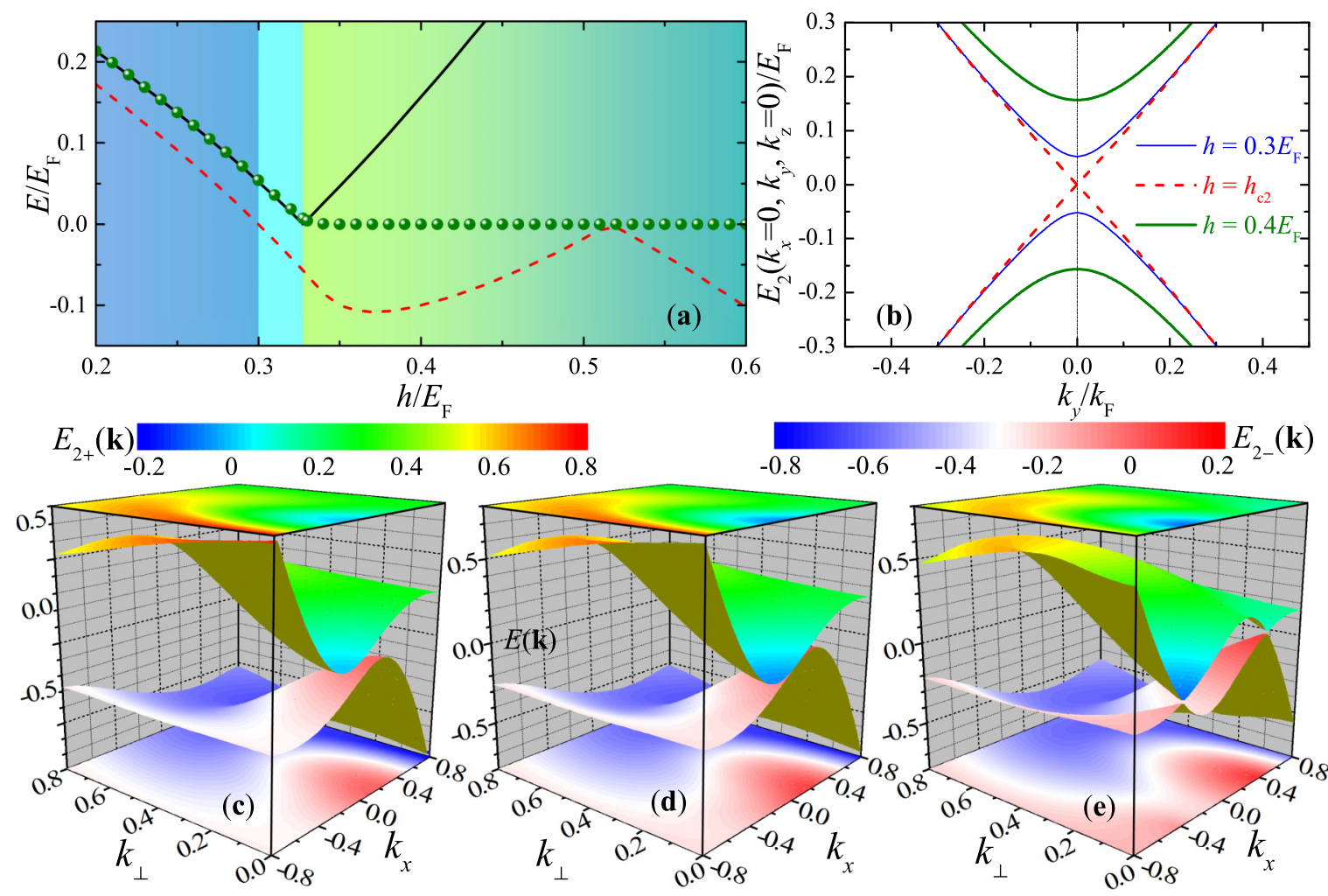

FIG. 3. (Color online) The evolution of the energy gap and of the topology of the Fermi surfaces at $\lambda=E_{F} / k_{F}$ with increasing in-plane Zeeman field. (a) The global energy gap $E_{g}=\min E_{2+}(\mathbf{k})$ (red dashed line), the energy gap at $\mathbf{k}=\mathbf{0}$ (black solid line), and the minimum energy of the surface states (green solid circles) when an open boundary is imposed on the $y-z$ plane. (b) The energy dispersion $E_{2 \pm}\left(k_{y}\right)$ at $k_{x}=0$ and $k_{z}=0$. (c), (d), and (e) The 3D full plot of the energy dispersion $E_{2 \pm}\left(k_{x}, k_{\perp}=\sqrt{k_{y}^{2}+k_{z}^{2}}\right)$ at $h_{c 1} \simeq 0.3 E_{F}(\mathrm{c}), h_{c 2} \simeq 0.327 E_{F}(\mathrm{~d})$, and $h=0.4 E_{F}$ (e). The wave vector and the energy are in units of $k_{F}$ and $E_{F}$, respectively. 
for the appearance of an SLTD topological superfluid when an out-of-plane Zeeman field is applied [8,9]. It is interesting that the gapless transition always occurs before the topological transition, as a result of $E_{g} \leqslant E_{2+}(\mathbf{k}=\mathbf{0})$. Thus, bulkgappedtopological FF superfluids, if they exist, must appear at a very high in-plane Zeeman field. As a superfluid analog of strong 3D topological insulators [1,2], they are anticipated to have the unique feature of a single Dirac cone for the energy dispersion of the Majorana edge states. Unfortunately, in the parameter space that we considered, we do not find their existence.

At the coupling strength $\lambda=E_{F} / k_{F}$, the gapless transition and the topological transition occur at $h_{c 1} \simeq 0.3 E_{F}$ and $h_{c 2} \simeq 0.327 E_{F}$, respectively, as can be seen from Fig. 3(a), where $E_{g}$ (red dashed line) and $E_{2+}(\mathbf{k}=\mathbf{0})$ (black solid line) become zero as the in-plane Zeeman field increases. When $h>h_{c 1}$, nodal points that satisfy $E_{2 \pm}(\mathbf{k})=0$ develop and form two closed surfaces in momentum space $[25,26]$. When the in-plane field increases further, passing through the threshold $h_{c 2}$ for the topological transition [see Fig. 3(d)], the energy dispersions of the particle and hole branches touch at two specific points $\left( \pm k_{W}, 0,0\right)$, as shown in Fig. 3(e). Around these points, the dispersion of Bogoliubov quasiparticles in the bulk acquires a linear structure and they thereby form a Dirac cone. This is precisely the energy dispersion for massless Weyl fermions [44-46]. In this sense, the gapless topological FF superfluid predicted in this work provides an avenue for the observation of Weyl fermions around the Weyl nodes $\left( \pm k_{W}, 0,0\right)$. Indeed, Weyl fermions have recently been discussed in the context of 3D gapped topological superfluids [47-49].

In our case, the appearance of the Weyl nodes and of the topological order is closely related. Due to the asymmetry in the $k_{x}$ axis, only one of the Weyl nodes is occupied. Thus, we may characterize the topological order of the gapless FF superfluid by using the topological invariant of Weyl fermions [2,50]:

$$
N_{W}=\int \frac{d^{3} \mathbf{k}}{24 \pi^{2}} \epsilon^{\mu \nu \rho} \operatorname{Tr}\left[Q_{\mathbf{k}}^{\dagger} \partial_{\mu} Q_{\mathbf{k}} Q_{\mathbf{k}}^{\dagger} \partial_{\nu} Q_{\mathbf{k}} Q_{\mathbf{k}}^{\dagger} \partial_{\rho} Q_{\mathbf{k}}\right],
$$

where $Q_{\mathbf{k}}$ is the unitary matrix determined by the BdG Hamiltonian, $\mu, v, \rho=\left(k_{x}, k_{y}, k_{z}\right)$, and the domain of the integration includes the isolated, occupied at zero temperature Weyl node. The gapless topological FF superfluid is characterized by a nonzero topological invariant $N_{W}= \pm 1$ [47,49], where the sign depends on the position of the Weyl nodes (i.e., $k_{x}=+k_{W}$ or $k_{x}=-k_{W}$ ). We note that the Weyl nodes always come in pairs.

\section{B. Majorana edge states}

To further demonstrate the topological nature of the gapless FF superfluid, we calculate the energy dispersion in the presence of an open boundary by imposing cylindrical hardwall confinement perpendicular to the $y-z$ plane with radius $L$ and with the $x$ axis being the symmetry axis (see Appendix B). There is a pair of zero-energy Majorana fermion states on the boundary of $r=\sqrt{y^{2}+z^{2}}=L$, which is the direct signature of a topologically nontrivial state. The existence of Majorana fermions is reported in Fig. 3(a) by green solid circles. They

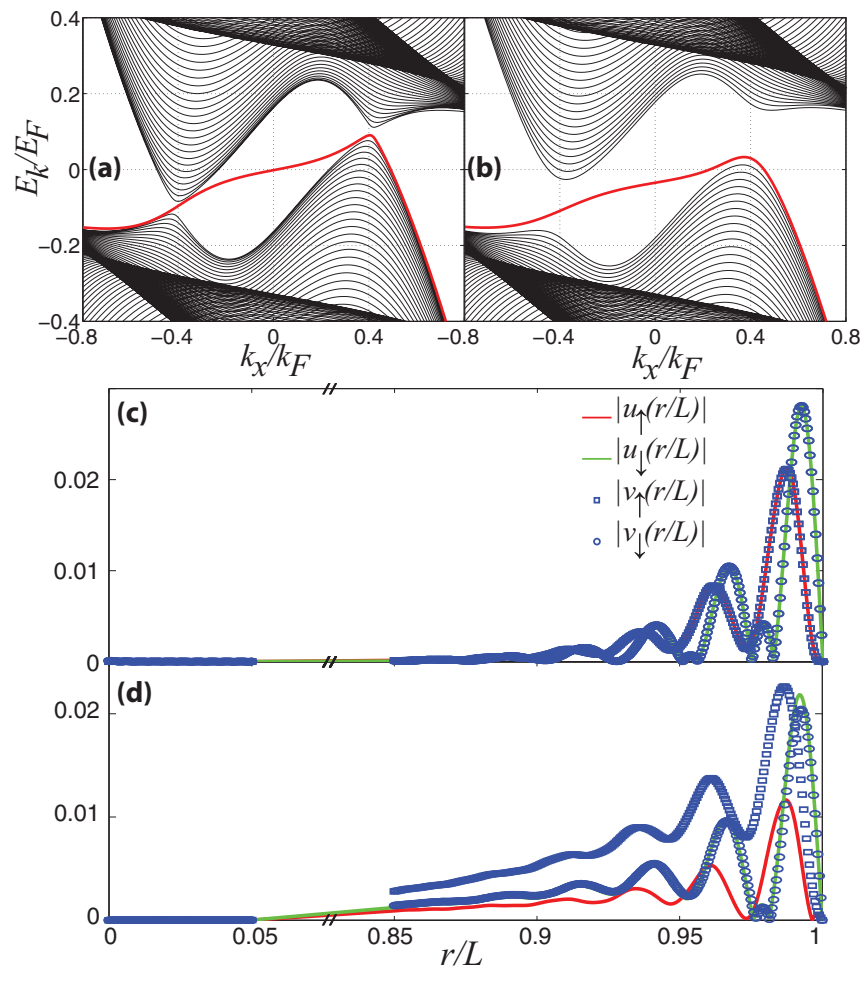

FIG. 4. (Color online) Majorana surface states arising from the hard-wall confinement perpendicular to the $y$ - $z$ plane. (a) and (b) The energy spectrum $E_{k_{x}}^{(m)}$ as a function of $k_{x}$ for $m=0$ and 10 , respectively. (c) The wave function of the zero-energy Majorana fermions for $m=0$, satisfying the symmetry $u_{\sigma}(r)=e^{i \vartheta} v_{\sigma}^{*}(r)$, where $\vartheta$ is a constant phase factor and $\sigma=\uparrow, \downarrow$. (d) The wave function of the zero-energy surface state for $m=10$. In numerical calculations, we have set the radius of the confinement $L=200 k_{F}^{-1}$. Other parameters are $\lambda=E_{F} / k_{F}$ and $h=0.4 E_{F}$. The wave function is normalized to unity: $\int_{0}^{L} 2 \pi r d r \sum_{\sigma}\left[\left|u_{\sigma}(r)\right|^{2}+\left|v_{\sigma}(r)\right|^{2}\right]=1$.

immediately appear after the change in the topology of the Fermi surfaces.

We now discuss in more detail the Majorana surface states, whose dispersion is shown in Fig. 4. Because the boundary we impose has cylindrical symmetry in the $y-z$ plane and translational symmetry along the $x$ direction, the quasiparticle wave function takes the following form:

$$
\Phi_{k_{x} \eta}^{\nu}=e^{i k_{x} x} e^{i m \phi}\left[u_{\eta \uparrow}^{v}(r), u_{\eta \downarrow}^{\nu}(r) e^{i \phi}, v_{\eta \uparrow}^{v}(r) e^{i \phi}, v_{\eta \downarrow}^{\nu}(r)\right]^{T},
$$

where $(x, r, \phi)$ form the cylindrical coordinates. States with different orbital angular momentum quantum number $m$ and linear momentum $k_{x}$ are decoupled; see Appendix B for more details. In Figs. 4(a) and 4(b), we plot the energy spectrum along the $k_{x}$ axis for $m=0$ and 10, respectively. The Majorana zero-energy mode can be identified by the energy crossing of the surface state contribution, at which points quasiparticle wave functions become localized near the boundary $r=L$, as shown in Figs. 4(c) and 4(d). The surface states smoothly connect to the Weyl nodes located approximately at $k_{W} \simeq 0.4 k_{F}$ in the bulk. As $|m|$ increases, the localization of the zero-energy surface modes deteriorates due to their hybridization with the bulk modes, and the desired symmetry $u_{\sigma}(r)=e^{i \vartheta} v_{\sigma}^{*}(r)(\sigma=\uparrow, \downarrow)$ for Majorana mode 
[51] is violated [see Fig. 4(d)]. One can prove that, as a result of particle-hole symmetry of the BdG equation, when $m \rightarrow-m-1$, and $k_{x} \rightarrow-k_{x}$, we have $E_{\eta v} \rightarrow-E_{\eta v}$. Hence for every zero-energy state at given $m$ and $k_{x}$, there is a corresponding zero-energy state at $-m-1$ and $-k_{x}$, which describes the same physical state. As a result, it is easy to see from Figs. 4(a) and 4(b) that for arbitrary azimuthal angular momentum $m$, the Majorana surface states have nearly the same unidirectional velocity $v\left(k_{x}\right)=\partial E_{k_{x}}^{(m)} / \partial k_{x}>0$. As there are no net atomic currents at equilibrium, the current carried by these copropagating surface states must be compensated by the current induced by some extra counterpropagating modes in the bulk. This is only possible when the system is gapless in the bulk, consistent with the gapless nature of our topological FF superfluid. The unidirectional surface states discussed in our work are therefore a unique feature of the gapless topological FF superfluid.

We may also consider imposing hard-wall confinement along a specific direction, for example adding two walls perpendicular to the $y$ axis at $y=0, L$. In this case, unidirectional Majorana surface states propagate in the same direction on opposite boundaries at $y=0$ and $y=L$, respectively. For detailed discussions, we refer to Appendix C.

It is worth noting that the $3 \mathrm{D}$ gapless topological $\mathrm{FF}$ superfluid cannot be viewed as a stack of 2D topological superfluids along a specific direction (i.e., the $x$ axis), unlike the standard 3D topological superfluids known so far. For the latter, the Majorana surface states of the 3D system can be understood as the edge states of the 2D system on the surfaces, which are parallel to the $x$ axis and therefore have a flat dispersion that does not depend on $k_{x}$ [48]. This is analogous to the trivial or weak 3D topological insulators [1,2]. In our case, due to the existence of spin-orbit coupling in all three spatial directions, the dispersion of the Majorana surface states is no longer flat. In this respect, the gapless topological FF superfluid might be better regarded as the superfluid analog of a strong topological insulator [1,2], although the surface states may not have a Dirac-cone-like dispersion due to the gapless bulk.

\section{Experimental accessibility}

Due to their significant parameter space in the phase diagram, our proposed gapless topological FF superfluids could be easily detected in current cold-atom experiments, where the isotropic or Rashba-type spin-orbit coupling can be engineered by using Raman lasers [40] or a sequence of pulsed inhomogeneous magnetic fields [41]. The unidirectional Majorana surface modes, as unique experimental evidence of the topological superfluid, could in principle be directly visible as arcs in momentum space at the Fermi surfaces in the momentum-resolved radiofrequency spectroscopy [51].

To give some typical numbers for a possible cold-atom experiment, we consider a strongly interacting Fermi cloud of $N=2 \times 10^{5}{ }^{40} \mathrm{~K}$ atoms confined in a harmonic trap with a geometric mean trapping frequency $\bar{\omega} \simeq 2 \pi \times 130 \mathrm{~Hz}$ [38]. The Fermi energy or Fermi temperature is about $T_{F}=(3 N)^{1 / 3} \hbar \bar{\omega} / k_{B} \simeq 500 \mathrm{nK}$. Experimentally, the coupling strength of the isotropic spin-orbit coupling and the Zeeman field may be independently tuned at will [40-42], according to the reported experiments with a Raman scheme for spin- orbit coupling [36-38]. Thus, the parameter space of the gapless topological phase could be easily reached. Actually, the major experimental difficulty is the realization of Fermi superfluidity in the presence of spin-orbit coupling. For the gapless topological phase shown in Fig. 2 at the interaction strength $1 /\left(k_{F} a_{s}\right)=-0.5$, we estimate that the superfluid transition temperature would be at about $T_{c} \simeq 0.1 T_{F} \simeq 50 \mathrm{nK}$. This temperature is known to be accessible in the absence of spin-orbit coupling [52].

In solid-state systems, a promising candidate for realizing the gapless topological FF superfluid is to use a quantum well with large Rashba and Dresselhaus spin-orbit couplings (i.e., hole-doped $\mathrm{InSb}$ ) in proximity to a conventional $s$ wave superconductor, where the in-plane Zeeman field in the quantum-well layer can be controlled to minimize the orbital effect [39]. Other candidates include noncentrosymmetric superconductors such as $\mathrm{CePt}_{3} \mathrm{Si}$ and $\mathrm{Li}_{2} \mathrm{Pd}_{x} P t_{3-x} \mathrm{~B}$ [53] in the presence of a magnetic field on the plane of Rashba spin-orbit coupling.

\section{CONCLUSIONS}

In summary, we have proposed a mechanism to create topologically nontrivial states by using an in-plane Zeeman field only. Interesting Gapless topological superfluids with an inhomogeneous Fulde-Ferrell pairing order parameter can be realized using three-dimensional spin-orbit-coupled $s$-wave superfluids, where the finite momentum pairing and topological order are both driven by the in-plane Zeeman field. They feature unidirectional surface modes and a pair of zero-energy Majorana fermions at the edges, which are quite different from the standard gapped topological superfluids that are known to date. These features will greatly enrich our understanding of topological quantum matter, in both solid-state and cold-atom systems.

We finally note that although the gapless topological superfluid has a gapless spectrum in the bulk, it shares the same stability as the standard gapped topological superfluid subject to disordered potentials. This is because, in momentum space, Majorana surface states - as the manifestation of topological order-are protected by the local energy gap at $\mathbf{k}=\mathbf{0}$, while in real space, the overlap between the zero-energy Majorana modes and the gapless bulk states is exponentially small. Therefore, we conclude that the predicted gapless topological superfluid should have potential applications similar to any gapped topological superfluid, in topological quantum information and quantum computation.

\section{ACKNOWLEDGMENTS}

We are grateful to Ming Gong for useful discussions. H.H. and X.J.L. were supported by the Australian Research Council (ARC) (Grants No. FT130100815, No. DP140103231, No. FT140100003 and No. DP140100637) and the National Key Basic Research Special Foundation of China (NKBRSFCChina) (Grant No. 2011CB921502). H.P. was supported by the NSF, the Welch Foundation (Grant No. C-1669), and the DARPA OLE program. 


\section{APPENDIX A: MEAN-FIELD BOGOLIUBOV-DE GENNES EQUATIONS}

The details of our theoretical framework have been presented in previous work [25,26]. Here we give a brief summary. Taking the mean-field approximation for the pairing interaction term, the model Hamiltonian of the Fermi system can be rewritten in a compact form, $\mathcal{H}=(1 / 2) \int d \mathbf{r} \Phi^{\dagger}(\mathbf{r}) \mathcal{H}_{\mathrm{BdG}}(\hat{\mathbf{k}}) \Phi(\mathbf{r})-V \Delta^{2} / U_{0}+$ $\sum_{\mathbf{k}}\left(\xi_{\mathbf{k}+\mathbf{Q} / 2}+\xi_{\mathbf{k}-\mathbf{Q} / 2}\right) / 2$, where the explicit form of $\mathcal{H}_{\mathrm{BdG}}(\hat{\mathbf{k}})$ in Eq. (2) is given by

$$
\left[\begin{array}{cccc}
\hat{\xi}_{\mathbf{k}+}+\lambda \hat{k}_{z} & \Lambda_{\mathbf{k}+}^{\dagger} & 0 & -\Delta \\
\Lambda_{\mathbf{k}+} & \hat{\xi}_{\mathbf{k}+}-\lambda \hat{k}_{z} & \Delta & 0 \\
0 & \Delta & -\hat{\xi}_{\mathbf{k}-}+\lambda \hat{k}_{z} & \Lambda_{\mathbf{k}-} \\
-\Delta & 0 & \Lambda_{\mathbf{k}-}^{\dagger} & -\hat{\xi}_{\mathbf{k}-}-\lambda \hat{k}_{z}
\end{array}\right]
$$

with $\quad \hat{\xi}_{\mathbf{k} \pm} \equiv \hbar^{2}(\hat{\mathbf{k}} \pm \mathbf{Q} / 2)^{2} /(2 m)-\mu \quad$ and $\quad \Lambda_{\mathbf{k} \pm} \equiv \lambda\left(\hat{k}_{x} \pm\right.$ $\left.q / 2+i \hat{k}_{y}\right) \pm h$. For a homogeneous Fermi gas with open boundary condition, the BdG Hamiltonian can be diagonalized by replacing the momentum operators $\hat{k}_{i}(i=x, y, z)$ by the corresponding $c$ numbers $k_{i}$. Thus, we obtain the energy spectrum of Bogoliubov quasiparticles $E_{\eta v}(\mathbf{k})$, where $v \in(+,-)$ denotes the particle or hole branch and $\eta \in(1,2)$ stands for the upper or lower band. The mean-field thermodynamic potential $\Omega_{\mathrm{mf}}$ at temperature $T$ can be written down straightforwardly,

$$
\begin{aligned}
\frac{\Omega_{\mathrm{mf}}}{V}= & \frac{1}{2 V} \sum_{\mathbf{k}}\left[\xi_{\mathbf{k}+\mathbf{Q} / 2}+\xi_{\mathbf{k}-\mathbf{Q} / 2}-\sum_{\eta=1,2} E_{\eta+}(\mathbf{k})\right] \\
& -\frac{\Delta^{2}}{U_{0}}-\frac{k_{B} T}{V} \sum_{\mathbf{k} \eta=1,2} \ln \left[1+e^{-E_{\eta+}(\mathbf{k}) / k_{B} T}\right],
\end{aligned}
$$

where the last term is the standard expression of thermodynamic potential for noninteracting Bogoliubov quasiparticles. Due to the inherent particle-hole symmetry in the Nambu spinor representation, the summation over the quasiparticle energy has been restricted to the particle branch to avoid double counting. For a given set of parameters (i.e., temperature $T, s$ wave scattering length $a_{s}$, etc.), different mean-field phases can be determined using the self-consistent stationary conditions, $\partial \Omega_{\mathrm{mf}} / \partial \Delta=0, \partial \Omega_{\mathrm{mf}} / \partial q=0$, as well as the conservation of the total atom number, $n=-(1 / V) \partial \Omega / \partial \mu$, where $n$ is the number density. At a given temperature, the ground state has the lowest free energy $F=\Omega+\mu N$. For simplicity, we only report the results at zero temperature. For a more quantitative description, in future studies strong pair fluctuations beyond mean field may be taken into account by using many-body $T$-matrix theories [54-56].

\section{APPENDIX B: MAJORANA SURFACE STATES WITH A CYLINDRICAL HARD-WALL CONFINEMENT}

To determine the Majorana surface states in the topologically nontrivial phase, we impose a cylindrical hard-wall potential, for example perpendicular to the $y-z$ plane, so that any single-particle wave function must vanish identically at the boundary $r=L$. We assume that the radius is sufficiently large so that we can use the solution of a uniform pairing gap.
Accordingly, in the BdG Hamiltonian Eq. (A1), we replace the momentum operator $k_{y}$ and $k_{z}$ with its corresponding derivatives in cylindrical coordinates, where the longitudinal axis is chosen along the $x$ direction. It can be diagonalized by using the following ansatz for the Bogoliubov wave functions:

$$
\left[\begin{array}{c}
u_{\uparrow}(\mathbf{r}) \\
u_{\downarrow}(\mathbf{r}) \\
v_{\uparrow}(\mathbf{r}) \\
v_{\downarrow}(\mathbf{r})
\end{array}\right]=\frac{e^{i m \theta}}{\sqrt{2 \pi}} \sum_{n=1}^{N_{\max }}\left[\begin{array}{c}
\frac{J_{m}\left(\kappa_{n}^{(m)} r / L\right)}{\sqrt{\mathcal{N}_{n}^{(m)}}} u_{n \uparrow} \\
\frac{J_{m+1}\left(\kappa_{n}^{(m+1)} r / L\right) e^{i \theta}}{\sqrt{\mathcal{N}_{n}^{(m+1)}}} u_{n \downarrow} \\
\frac{J_{m+1}\left(\kappa_{n}^{(m+1)} r / L\right) e^{i \theta}}{\sqrt{\mathcal{N}_{n}^{(m+1)}}} v_{n \uparrow} \\
\frac{J_{m}\left(\kappa_{n}^{(m)} r / L\right)}{\sqrt{\mathcal{N}_{n}^{(m)}}} v_{n \downarrow}
\end{array}\right] e^{i k_{x} x},
$$

where $\kappa_{n}^{(m)}$ is the $n$th positive root of the Bessel function of the first kind $J_{m}(\rho)$ with $m \geqslant 0$. For states with $m<$ 0 , we have instead $J_{-m}(\rho)=(-1)^{m} J_{m}(\rho)$. The orthogonal condition is given by $\int_{0}^{L} J_{m}\left(\kappa_{n}^{(m)} r / L\right) J_{m}\left(\kappa_{l}^{(m)} r / L\right) r d r=$ 0 , where integer $n \neq l$ and normalization reads $\mathcal{N}_{n}^{(m)}=$ $\int_{0}^{L} J_{m}\left(\kappa_{n}^{(m)} r / L\right) J_{m}\left(\kappa_{n}^{(m)} r / L\right) r d r=\frac{1}{2} L^{2}\left[J_{m+1}\left(\kappa_{n}^{(m)}\right)\right]^{2}$. Inserting this ansatz into the $\mathrm{BdG}$ equation, we convert the $\mathrm{BdG}$ Hamiltonian into a $4 N_{\max }$ by $4 N_{\max }$ Hermitian matrix,

$$
\begin{gathered}
\mathcal{H}_{11 n}^{(m)} u_{n \uparrow}-i \lambda \sum_{l=1}^{N_{\max }} \mathcal{W}_{l n}^{(m)} u_{l \downarrow}-\Delta v_{n \downarrow}=E_{k_{x}}^{(m)} u_{n \uparrow}, \\
i \lambda \sum_{l=1}^{N_{\max }} \mathcal{W}_{n l}^{(m)} u_{l \uparrow}+\mathcal{H}_{22 n}^{(m)} u_{n \downarrow}+\Delta v_{n \uparrow}=E_{k_{x}}^{(m)} u_{n \downarrow}, \\
\Delta u_{n \downarrow}+\mathcal{H}_{33 n}^{(m)} v_{n \uparrow}+i \lambda \sum_{l=1}^{N_{\max }} \mathcal{W}_{n l}^{(m)} v_{l \downarrow}=E_{k_{x}}^{(m)} v_{n \uparrow}, \\
-\Delta u_{n \uparrow}-i \lambda \sum_{l=1}^{N_{\max }} \mathcal{W}_{l n}^{(m)} v_{l \uparrow}+\mathcal{H}_{44 n}^{(m)} v_{n \downarrow}=E_{k_{x}}^{(m)} v_{n \downarrow},
\end{gathered}
$$

where all the matrix elements have been analytically worked out (not shown here). The diagonalization directly gives rise to the energies and wave functions of the Majorana surface states.

\section{APPENDIX C: MAJORANA SURFACE STATES WITH A HARD-WALL CONFINEMENT ALONG THE $y$ DIRECTION}

Here we discuss the Majorana surface states with a hard-wall potential along a specific direction, say along the $y$ direction. Any single-particle wave function must vanish identically at the boundary $y=0$ or $y=L$. We assume that the length $L$ is sufficiently large, so we use the solution of a uniform pairing gap. Accordingly, in the BdG Hamiltonian Eq. (A1), we replace the momentum operator $k_{y}$ with $-i \partial_{y}$. It can be diagonalized by using the following ansatz for the Bogoliubov wave functions:

$$
\begin{aligned}
& u_{\sigma}(y)=\sum_{n=1}^{N_{\max }} u_{n \sigma} \psi_{n}(y), \\
& v_{\sigma}(y)=\sum_{n=1}^{N_{\max }} v_{n \sigma} \psi_{n}(y),
\end{aligned}
$$



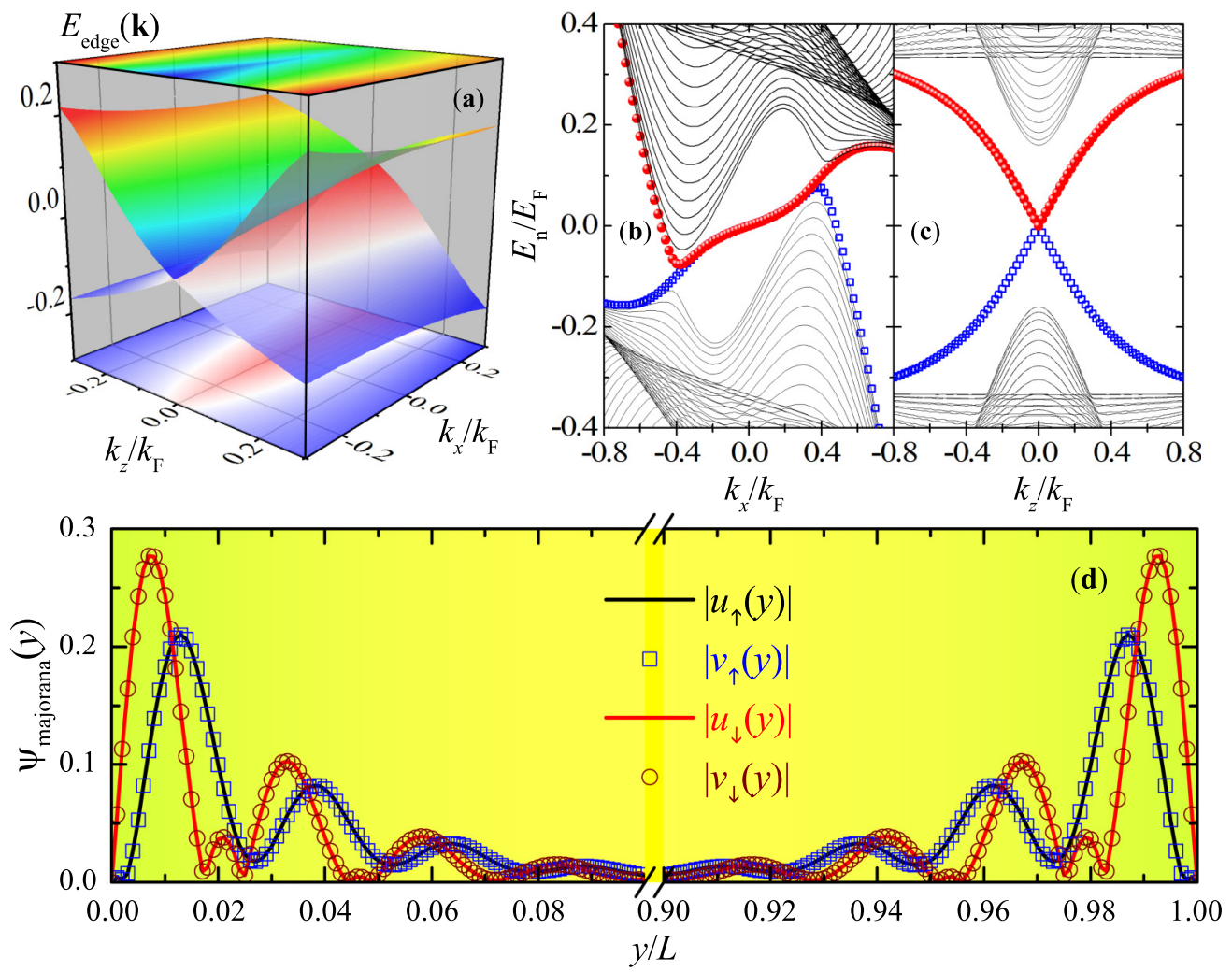

FIG. 5. (Color online) Majorana surface states arising from the hard-wall confinement along the $y$ direction. (a) The surface-state dispersion forms two sheets that cross at the line $k_{z}=0$. (b) and (c) The full energy spectrum $E_{2 \pm}\left(k_{x}, k_{z}\right)$ along the $k_{z}$ or $k_{x}$ axis, respectively. The surface states at the two boundaries are highlighted by red solid circles and blue empty squares, respectively. (d) The wave function of the zero-energy Majorana fermions at $k_{x}=0$ and $k_{z}=0$. In numerical calculations, we have set the length of the confinement $L=200 k_{F}^{-1}$. Other parameters are $\lambda=E_{F} / k_{F}$ and $h=0.4 E_{F}$ as in Fig. 4. The wave function is normalized to unity: $\int_{0}^{L} d y \sum_{\sigma}\left[\left|u_{\sigma}(y)\right|^{2}+\left|v_{\sigma}(y)\right|^{2}\right]=1$.

where $\psi_{n}(y)=\sqrt{2 / L} \sin [n \pi y / L]$ is the eigenfunction of the hard-wall potential with eigenvalue $\epsilon_{n}=\hbar^{2} n^{2} \pi^{2} /\left(2 m L^{2}\right)$. Inserting this ansatz into the $\mathrm{BdG}$ equation, we convert the BdG Hamiltonian into a $4 N_{\max }$ by $4 N_{\max }$ symmetric matrix, whose diagonalization leads directly to the energies and wave functions of the Majorana surface states.

With this hard-wall confinement, the dispersion of Majorana surface states is shown in Fig. 5. In momentum space, $k_{x}$ and $k_{z}$ are still good quantum numbers, so we actually plot $\min E_{2+}\left(k_{x}, k_{z}\right)$ and $\max E_{2-}\left(k_{x}, k_{z}\right)$. There are two sheets in the energy dispersion [Fig. 5(a)], corresponding to the surface states localized at the boundary $y=0$ and $y=L$, respectively. Remarkably, these two sheets cross at the line $k_{z}=0$, indicating that along this line the two branches of surface states are unidirectional, that is, propagating in the same direction on opposite boundaries. This is highlighted in Fig. 5(b), from which we also identify that the unidirectional Majorana surface states smoothly connect the two Weyl nodes $\left( \pm k_{W}, 0,0\right)$ in the bulk, where $k_{W} \simeq 0.4 k_{F}$. Recall that at equilibrium there are no net atomic currents. As in the cylindrically symmetric case, the current due to these copropagating surface states on opposite boundaries therefore must be compensated by the current induced by some extra counterpropagating modes in the bulk. This can only happen in systems with a gapless bulk. We note that the unidirectional Majorana surface states only occur along the line $k_{z}=0$. Actually, if we make a cut on the two sheets along other directions, for example along the line $k_{x}=0$, it is easy to see that the Majorana surface states become counterpropagating [see Fig. 5(c) for the dispersion as a function of $k_{z}$ ], resembling the surface states in the standard gapped topological superfluid. This follows the fact that at $k_{x}=0$ our topological FF superfluid is actually gapped in the bulk. By comparing the two limiting cases shown in Figs. 5(b) and 5(c), it is clear that the unidirectional surface states discussed in our work are a unique feature of gapless topological FF superfluid.
[1] M. Z. Hasan and C. L. Kane, Rev. Mod. Phys. 82, 3045 (2010).

[2] X.-L. Qi and S.-C. Zhang, Rev. Mod. Phys. 83, 1057 (2011).

[3] C. Nayak, C. H. Simon, A. Stern, M. Freedman, and S. Das Sarma, Rev. Mod. Phys. 80, 1083 (2008).

[4] F. Wilczek, Nat. Phys. 5, 614 (2009).
[5] N. Read and D. Green, Phys. Rev. B 61, 10267 (2000).

[6] G. Moore and N. Read, Nucl. Phys. B 360, 362 (1991).

[7] L. Fu and C. L. Kane, Phys. Rev. Lett. 100, 096407 (2008).

[8] J. D. Sau, R. M. Lutchyn, S. Tewari, and S. Das Sarma, Phys. Rev. Lett. 104, 040502 (2010). 
[9] Y. Oreg, G. Refael, and F. von Oppen, Phys. Rev. Lett. 105, 177002 (2010).

[10] C. Zhang, S. Tewari, R. M. Lutchyn, and S. Das Sarma, Phys. Rev. Lett. 101, 160401 (2008).

[11] M. Sato, Y. Takahashi, and S. Fujimoto, Phys. Rev. Lett. 103, 020401 (2009).

[12] V. Mourik, K. Zuo, S. M. Frolov, S. R. Plissard, E. P. A. M. Bakkers, and L. P. Kouwenhoven, Science 336, 1003 (2012).

[13] J. R. Williams, A. J. Bestwick, P. Gallagher, S. S. Hong, Y. Cui, A. S. Bleich, J. G. Analytis, I. R. Fisher, and D. GoldhaberGordon, Phys. Rev. Lett. 109, 056803 (2012).

[14] L. P. Rokhinson, X. Liu, and J. K. Furdyna, Nat. Phys. 8, 795 (2012).

[15] L. Dong, L. Jiang, H. Hu, and H. Pu, Phys. Rev. A 87, 043616 (2013).

[16] V. B. Shenoy, Phys. Rev. A 88, 033609 (2013).

[17] V. Barzykin and L. P. Gor'kov, Phys. Rev. Lett. 89, 227002 (2002).

[18] S. K. Yip, Phys. Rev. B 65, 144508 (2002).

[19] D. F. Agterberg and R. P. Kaur, Phys. Rev. B 75, 064511 (2007).

[20] O. Dimitrova and M. V. Feigel'man, Phys. Rev. B 76, 014522 (2007).

[21] K. Michaeli, A. C. Potter, and P. A. Lee, Phys. Rev. Lett. 108, 117003 (2012).

[22] Z. Zheng, M. Gong, X. Zou, C. Zhang, and G.-C. Guo, Phys. Rev. A 87, 031602(R) (2013).

[23] F. Wu, G.-C. Guo, W. Zhang, and W. Yi, Phys. Rev. Lett. 110, 110401 (2013).

[24] X.-J. Liu and H. Hu, Phys. Rev. A 87, 051608(R) (2013).

[25] L. Dong, L. Jiang, and H. Pu, New J. Phys. 15, 075014 (2013).

[26] X.-F. Zhou, G.-C. Guo, W. Zhang, and W. Yi, Phys. Rev. A 87, 063606 (2013).

[27] H. Hu and X.-J. Liu, New J. Phys. 15, 093037 (2013).

[28] C. Chen, Phys. Rev. Lett. 111, 235302 (2013).

[29] X.-J. Liu and H. Hu, Phys. Rev. A 88, 023622 (2013).

[30] C. Qu, Z. Zheng, M. Gong, Y. Xu, L. Mao, X. Zou, G.-C. Guo, and C. Zhang, Nat. Commun. 4, 2710 (2013).

[31] W. Zhang and W. Yi, Nat. Commun. 4, 2711 (2013).

[32] C. F. Chan and M. Gong, Phys. Rev. B 89, 174501 (2014).

[33] Y. Cao, S.-H. Zou, X.-J. Liu, S. Yi, G.-L. Long, and H. Hu, Phys. Rev. Lett. 113, 115302 (2014).
[34] P. Fulde and R. A. Ferrell, Phys. Rev. 135, A550 (1964).

[35] Y.-J. Lin, K. Jiménez-García, and I. B. Spielman, Nature (London) 471, 83 (2011).

[36] P. Wang, Z.-Q. Yu, Z. Fu, J. Miao, L. Huang, S. Chai, H. Zhai, and J. Zhang, Phys. Rev. Lett. 109, 095301 (2012).

[37] L. W. Cheuk, A. T. Sommer, Z. Hadzibabic, T. Yefsah, W. S. Bakr, and M. W. Zwierlein, Phys. Rev. Lett. 109, 095302 (2012).

[38] Z. Fu, L. Huang, Z. Meng, P. Wang, X.-J. Liu, H. Pu, H. Hu, and J. Zhang, Phys. Rev. A 87, 053619 (2013).

[39] J. Alicea, Phys. Rev. B 81, 125318 (2010).

[40] B. M. Anderson, G. Juzeliūnas, V. M. Galitski, and I. B. Spielman, Phys. Rev. Lett. 108, 235301 (2012).

[41] B. M. Anderson, I. B. Spielman, and G. Juzeliūnas, Phys. Rev. Lett. 111, 125301 (2013).

[42] Z.-F. Xu, L. You, and M. Ueda, Phys. Rev. A 87, 063634 (2013).

[43] For the latest review, see, for example, S. K. Yip, Annu. Rev. Condens. Matter Phys. 5, 15 (2014), and reference therein.

[44] X. Wan, A. M. Turner, A. Vishwanath, and S. Y. Savrasov, Phys. Rev. B 83, 205101 (2011).

[45] A. A. Burkov and L. Balents, Phys. Rev. Lett. 107, 127205 (2011).

[46] G. Xu, H. Weng, Z. Wang, X. Dai, and Z. Fang, Phys. Rev. Lett. 107, 186806 (2011).

[47] M. Gong, S. Tewari, and C. Zhang, Phys. Rev. Lett. 107, 195303 (2011).

[48] J. D. Sau and S. Tewari, Phys. Rev. B 86, 104509 (2012).

[49] Y. Xu, R.-L. Chu, and C. Zhang, Phys. Rev. Lett. 112, 136402 (2014).

[50] A. P. Schnyder, S. Ryu, A. Furusaki, and A. W. W. Ludwig, Phys. Rev. B 78, 195125 (2008).

[51] X.-J. Liu, L. Jiang, H. Pu, and H. Hu, Phys. Rev. A 85, 021603(R) (2012).

[52] S. Giorgini, L. P. Pitaevskii, and S. Stringari, Rev. Mod. Phys. 80, 1215 (2008).

[53] A. P. Schnyder and S. Ryu, Phys. Rev. B 84, 060504(R) (2011).

[54] X.-J. Liu and H. Hu, Phys. Rev. A 72, 063613 (2005).

[55] H. Hu, X.-J. Liu, and P. D. Drummond, Europhys. Lett. 74, 574 (2006)

[56] H. Hu, X.-J. Liu, and P. D. Drummond, Phys. Rev. A 77, 061605 (2008). 